\title{
CIRCLE STRUCTURE DERIVED FROM DECOMPOSITION OF ASYMMETRIC DATA MATRIX
}

\author{
Takayuki Saito*
}

\begin{abstract}
Among many approaches to analysis of asymmetric data, this paper is concerned with the matrix decomposition approach. Focussing on Gower's procedure, we study conditions to derive circle structure from an asymmetric data matrix. Results are presented in terms of some theorems and lemmas, followed by discussion with numerical illustrations.
\end{abstract}

\section{Introduction}

\subsection{Background}

In data analysis in many desciplines, investigators are often concerned with a relationship between pairs of objects that is asymmetric. Let such a matrix be $O=\left(o_{j k}\right)$ where $o_{j k}$ indicates the measure of relationship and the diagonal entry may or may not be defined. Let us explain two examples (see other examples in Saito, 1997).

In a study of pecking behavior among a group of pigeons in animal psychology, $o_{j k}$ measures the frequency that individual $j$ pecks individual $k$, and the diagonal entry is undefined. In a study of spatial association of tree species in ecology, $o_{j k}$ measures the number of times that a tree of species $k$ is the nearest neighbour of species $j$, and the diagonal entry is defined.

If such asymmetry is considered a source of bias or due to a chance effect of sampling to be eliminated, one way of treating the data is to construct a symmetric matrix by taking the mean of $o_{j k}$ and $o_{k j}$ and apply procedures to analyze symmetric data. In the two examples above, however, the asymmetry is considerd to embody some structure contained in or some important information about the data. Then it is of interest to analyze the asymmetry.

Approaches to analysis of asymmetry from this standpoint can be classified into four major types: scaling (e.g. Bock and Jones, 1968; Chino, 1978; Weeks and Bentler, 1982; Harshman, 1978; Harshman et al., 1982; Saito, 1991, 1994; Sato, 1988, 1989), matrix decomposition (e.g. Gower, 1977; Constantine and Gower, 1978; Escoufier and Grorud, 1980; Chino and Shiraiwa, 1993), clustering (e.g. Ferligoj and Batagelj, 1983) and graph theory. The review of the methodological differences may be omitted here.

Although the first two approaches were originally suggested from different backgrounds, both are similarly used for spatial ordination of objects. Saito (1997) pointed out the advantage of the matrix decomposition approach over the scaling one because it is not concerned with model construction but with decomposition of a matrix in the context of

\footnotetext{
*Graduate School of Decision Science and Technology, Tokyo Institute of Technology, Ookayama 2-12-1, Meguro-ku, Tokyo 152-8552, Japan

Key words: Cyclic matrix; Gower's procedure; Line structure; Singular value decomposition; Skewsymmetric matrix
} 
linear algebra. According to Gower's and Escoufier and Grorud's procedures, one obtains a two-dimensional representation of points in terms of coordinates. If the data reveal a noticeable pattern such as line or circle in the representation, one would interpret it as a meaningful structure or significant information involved in the data. From this viewpoint, Saito (1997) studied conditions to obtain line structure from the asymmetric data matrix regarding the two procedures. The line structure means that all points are located on a line in the configuration.

\subsection{Purpose}

Taking the same viewpoint, we are interested in conditions to derive a circle structure from the asymmetric data matrix. Let us show an example which motivated us to do this study. The data are taken from Hobbs and Legg (1980) who dealt with the changes in vegetation of a region during regeneration after burning. In the data matrix $O=\left(o_{j k}\right)$, element $o_{j k}$ describes the number of transitions between preceding state $j$ and succeeding state $k$. Applying Gower's procedure to a skew-symmetric matrix constructed from $O$, we obtain a two-dimensional plot as displayed in Figure 1. In the plot we find a nearly circular pattern, and the clockwise order of states about the origin suggests a cyclical climax as expected in plant ecological studies.

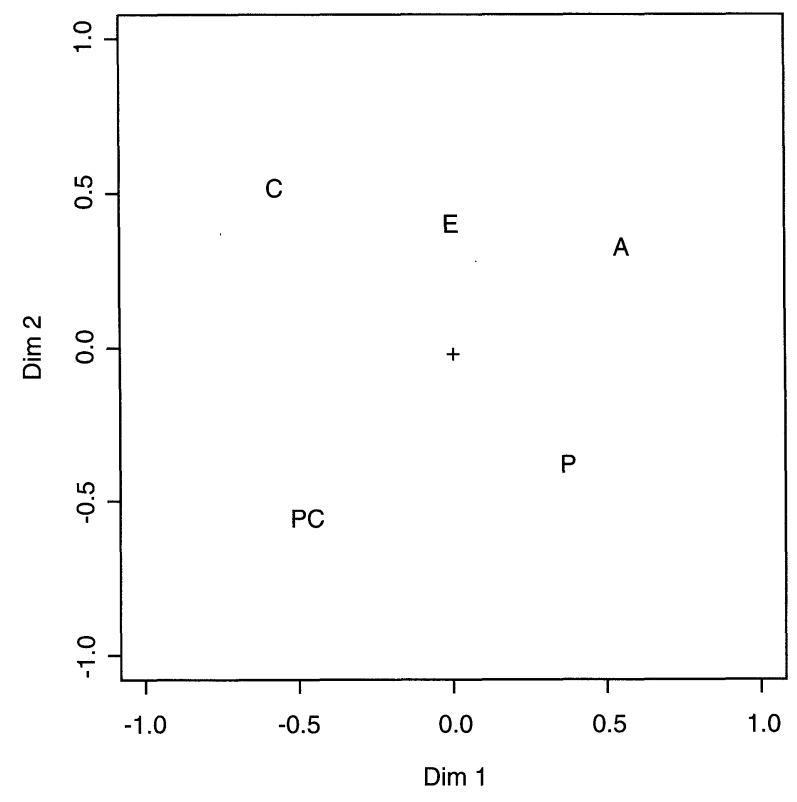

Fig. 1: The configuration of vegetation states 
The purpose of this paper, therefore, is to study some conditions to obtain circle structure from the asymmetric data matrix by using Gower's procedure. Here the circle structure means, in a descriptive sense, that all points are located on a circle in the plot on a twodimesional plane. This approach to argue such a structure is different from Chino and Shiraiwa's approach on which they argued the metric structure in a vector space associated with an Hermitian matrix.

In what follows, we first give a preliminary review for conveniences of exposition, and state clearly the difference of the two approaches. Next we consider those conditions in connection with a special type of matrix (cyclic or circulant matrix). Then we consider those conditions regarding an asymmetric matrix that is not necessarily cyclic. The results are presented as some theorems and lemmas, followed by discussion and remarks with numerical illustration.

\section{Preliminaries}

\subsection{Two types of approaches}

Denote an $n \times n$ asymmetric matrix by $O=\left(o_{j k}\right)$ for which $o_{j k} \neq o_{k j}$ in general and the diagonal entries are defined. Define a symmetric matrix $T$, a skew-symmetric matrix $S=\left(s_{j k}\right)$ and an Hermitian matrix $H$ as follows:

$$
\begin{aligned}
T & =\left(O+O^{\prime}\right) / 2 \\
S & =\left(O-O^{\prime}\right) / 2 \\
H & =T+i \cdot S,
\end{aligned}
$$

where $i^{2}=-1$. There are two different types of approaches to analyze matrix $O$. One is to treat $T$ and $S$ simultaneously, which leads to treating $H$ in a way. In this way, Escoufier and Grorud (1980) suggested a procedure to analyze $H$, and Chino and Shiraiwa (1993) generalized it in a unified study for a family of Hermitian form models (HFM). The other is dealing with $T$ and $S$ separately, as Gower suggested. Then the analysis of asymmetry is focused on the relationship in terms of skew-symmetry, such as

$$
s_{j k}+s_{k j}=0 .
$$

This approach is called skew-symmetry analysis (SSA). Let us review HFM in contrast to SSA brifely.

\subsection{HFM}

Chino and Shiraiwa argued geometric structures inherent in $H$ in the context of finite Hilbert space. They showed that if $H$ is positive (negative) semidefinite, then a metric tensor is defined in the space. In the present review, however, we are concerned with arguments that are followed in a simple context of complex-vector space with an inner product defined. Write the eigenequation of $H$ as

$$
H \boldsymbol{w}_{t}=\lambda_{t} \boldsymbol{w}_{t}
$$

The eigenvalue $\lambda_{t}$ is real, whereas $\boldsymbol{w}_{t}=\left(w_{j t}\right)$ is generally complex $(t=1,2, \ldots, n)$. The element is expressed as $w_{j t}=x_{j t}+i \cdot y_{j t}$. From (3) and (5), we note that

$$
\sum_{j=1}^{n} o_{j j}=\operatorname{tr} H=\sum_{t=1}^{n} \lambda_{t} .
$$


If all $o_{j j}=0$, then $\sum_{j=1}^{n} \lambda_{t}=0$, which implies that $H$ is indefinite. Therefore, when all the diagonal entries are zero, the metric tensor is not defined.

For simplicity, let $H$ be positive semidefinite $(p s d)$ with rank $r$, that is to say, $\lambda_{t}>$ $0(t=1,2, \cdots, r)$ and the remaining roots are all zero. Let $\Lambda=\operatorname{diag}\left(\lambda_{1}, \ldots, \lambda_{r}\right)$. Let $\widetilde{\boldsymbol{w}}_{j}=\left(w_{j 1}, \ldots, w_{j r}\right)^{\prime}$, and write its complex conjugate as $\widetilde{\boldsymbol{w}}_{j}^{*}$. Define an inner product and a norm in terms of the Hermitian product by

$$
\left(\widetilde{\boldsymbol{w}}_{j}, \widetilde{\boldsymbol{w}}_{k}\right)=\widetilde{\boldsymbol{w}}_{j}^{\prime} \Lambda \widetilde{\boldsymbol{w}}_{k}^{*} \quad \text { and } \quad\left\|\widetilde{\boldsymbol{w}}_{j}\right\|=\sqrt{\left(\widetilde{\boldsymbol{w}}_{j}, \widetilde{\boldsymbol{w}}_{j}\right)}
$$

Then the metric (distance) in terms of this norm is defined as

$$
d_{j k}=\left\|\widetilde{\boldsymbol{w}}_{j}-\widetilde{\boldsymbol{w}}_{k}\right\|
$$

By some manipulation, it is found that

$$
o_{j j}=\left\|\widetilde{\boldsymbol{w}}_{j}\right\|^{2}=\sum_{t=1}^{r} \lambda_{t}\left(x_{j t}^{2}+y_{j t}^{2}\right) .
$$

When $o_{j j}=0$, it implies that $\left\|\widetilde{\boldsymbol{w}}_{j}\right\|=0$. Thus, if the metric in terms of (7) and (8) is defined with the definite property of $H$, it is necessary that all $o_{j j}$ should be defined and be nonnegative and not all $o_{j j}=0$. Turn to the scale level of measurement. Entries of $H$ are invariant on a ratio scale but not on an interval scale. Then, the geometric structure derived from $H$ is variant under linear transformation of observations such that

$$
p_{j k}=\alpha o_{j k}+\beta
$$

When a double centralization is applied to a matrix of interval scaled data, it may give rise to a mixture of positive and negative values for the diagonal entries in $H\left(p_{j k}\right)$, possibly violating the necessary condition for being positive semidefinite.

According to Chino and Shiraiwa, when $H$ is psd with rank $r$, it is justified to examine the distance structure and symplectic stucture involved in the derived configuration. Given such a configutration, let us consider its utilization for practical data analysis. It is extremely difficult to detect any patterns to characterize those structures in the $r$-dimensional complex space. In such a difficult detection, one would have to examine symmetric $d_{j k}$ as well as asymmetric $\bar{d}_{j k}$ for every pair $(j, k)$ and more completely, would refer to

$$
o_{j k}=-\frac{1}{2}\left(d_{j k}^{2}+\bar{d}_{j k}^{2}\right)+d_{0 j}^{2}+d_{0 k}^{2}
$$

where $d_{j k}$ is defined by (8), and

$$
\bar{d}_{j k}=\left\|\widetilde{\boldsymbol{w}}_{j}-i \cdot \widetilde{\boldsymbol{w}}_{k}\right\| \quad \text { and } \quad d_{0 j}=\left\|\widetilde{\boldsymbol{w}}_{j}\right\| .
$$

When $r=1$ in particular, plotting $n$ objects in terms of $\left(x_{j}, y_{j}\right)$ on a real 2-dimensional plane, one may examine and interpret the structure fairly easily in terms of $d_{j k}, \bar{d}_{j k}$ and the oriented paralleotope associated with $s_{j k}$. When $r>1$, one would have to do a similar treatment for interpreting the configuration in a descriptive way, by making a plot in terms of $\left(x_{j t}, y_{j t}\right)$ for each $t(=1,2, \ldots, r)$. 


\subsection{SSA}

According to Gower's original suggestion, one applies singular value decomposition (SVD) to $S$. For analyzing asymmetry, however, SSA can be applied to skew-symmtric matrices that may be constructed from $O$ in different ways from (2) (Saito, 1997). Note that SSA is applicable for cases in which diagonal elements may or may not be defined. For a matrix with diagonal entries, one may construct a skew-symmetric matrix, for example, as

$$
\tilde{s}_{j k}=\left(o_{j k}-o_{k j}+o_{j j}-o_{k k}\right) / 2 .
$$

By SVD, we have a representation of $S$ as

$$
S=\sum_{k=1}^{m} \mu_{k}\left(\boldsymbol{u}_{k} \boldsymbol{v}_{k}^{\prime}-\boldsymbol{v}_{k} \boldsymbol{u}_{k}^{\prime}\right)
$$

Here $\boldsymbol{u}_{1}$ and $\boldsymbol{v}_{1}$ are singular vectors associated with singular value $\mu_{1}$, and $\boldsymbol{u}_{2}$ and $\boldsymbol{v}_{2}$ singular vectors associated with $\mu_{2}$ and so on, where $\mu_{k} \geq 0(k=1,2, \cdots, m=[n / 2])$ and numbered in descending order of magnitude. They are all orthogonal and normalized as

$$
\boldsymbol{u}_{p}^{\prime} \boldsymbol{u}_{q}=\boldsymbol{u}_{p}^{\prime} \boldsymbol{v}_{q}=\boldsymbol{v}_{p}^{\prime} \boldsymbol{u}_{q}=\boldsymbol{v}_{p}^{\prime} \boldsymbol{v}_{q}=\delta_{p q}
$$

where $\delta_{p q}$ is the Kronecker delta.

Take a pair of singular vectors $\boldsymbol{u}=\left(u_{j}\right)$ and $\boldsymbol{v}=\left(v_{j}\right)$. Define vectors $\widetilde{\boldsymbol{g}}_{j}=\left(u_{j}, v_{j}\right)^{\prime}$. Plotting $n$ objects with coordinates $\left(u_{j}, v_{j}\right)$, we have a configuration of $n$ points on the 2-dimensional plane. Under tranformation (10), it is noted that SVD (11) is invariant up to the scale unit $\alpha$, and so is the configuration derived by SSA. Let $\triangle_{o j k}$ express the triangle formed by the origin and points $j$ and $k$ in the plot. The area of $\triangle_{o j k}$ corresponds to $\left|s_{j k}\right|$ and skew-symmetry (4) represents the orientation. As Gower stated, symmetric distance is not defined with $s_{j k}$ in the configuration. It should be noted that this property does not eliminate referring to Euclidean distance in the configuration. However, if one would apply HFM to $S$, that does not make sense. Notice that it is not meaningful to refer to metric (8) on the basis of (7) because $\left\|\widetilde{\boldsymbol{w}}_{j}\right\|^{2}=s_{j j}=0$ by (9). This point is argued in Chino and Shiraiwa more mathematically, stating that a simplectic structure is associated with $S$.

Even considering the property, one may refer to the length of $\widetilde{\boldsymbol{g}}_{j}$. When the vectors are of the same length, the $n$ points are located on a circle. Then the degree of angle spanned by $\widetilde{\boldsymbol{g}}_{j}$ and $\widetilde{\boldsymbol{g}}_{k}$ is proportional to $\left|s_{j k}\right|$. We are interested in some conditions under which this sort of circle pattern appears in the plot. When such a pattern is found in the plot, we will say that the pair of singular vectors yields a circle structure in a descriptive sense. As in the case of applying the biplot technique (Gabriel, 1981), our referring to the length of $\widetilde{\boldsymbol{g}}_{j}$ and the circle structure is not concerned with metric (8) based on (7). In view of this point, we will argue the length of vectors in the two-dimensional plot in theorems presented later.

We summarize a comparison of SSA with HFM. Unlike HFM, there is no necesaary condition to perform SSA on $O$. It can be applied even to a data matrix for which diagonal entries are all zero or undefined. Linear transformation of data is permissible for SSA but not for HFM. An adavantage of HFM over SSA is its mathematical arguments with psd $H$ that one can work on symmetric $d_{j k}$, asymmetric $\bar{d}_{j k}$ and the oriented paralleotope simultaneously. For $r>1$, however, it is difficult to utilize those characteristics in practical applications to a good extent. Thus SSA still remains as a useful tool for data analysis. 


\subsection{Cyclic matrix}

An $n \times n$ asymmetric matrix $C=\left(c_{j k}\right)$ is defined to be cyclic (or circulant) matrix if it satisfies

$$
c_{j k}=q_{n-j+k} \quad \text { where } q_{0}=q_{n} \quad \text { and } \quad q_{n+k}=q_{k} .
$$

It is explicitly stated as

$$
C=\left(\begin{array}{ccccc}
q_{0} & q_{1} & q_{2} & \ldots & q_{n-1} \\
q_{n-1} & q_{0} & q_{1} & \ldots & q_{n-2} \\
\vdots & \vdots & \vdots & \ddots & \vdots \\
q_{2} & q_{3} & q_{4} & \ldots & q_{1} \\
q_{1} & q_{2} & q_{3} & \ldots & q_{0}
\end{array}\right)
$$

Write the eigenproblem of $C$ as'

$$
C z=\lambda z
$$

The eigenvalue and eigenvector of this equation were provided, probably for the first time, by Berlin and Kac (1952) in connection with a physical problem. We will refer to their result as a theorem hereafter.

\section{Theorem (Berlin and Kac)}

The eigenvalue and eigenvector of (14) are provided as follows:

$$
\begin{aligned}
& \boldsymbol{z}=\left(1, \omega, \omega^{2}, \ldots, \omega^{n-1}\right) \\
& \lambda=q_{0}+q_{1} \omega+q_{2} \omega^{2}+\ldots+q_{n-1} \omega^{n-1} .
\end{aligned}
$$

Here $\omega=e^{i \theta}$ with $\theta=2 \pi / n$, which is a root of unity, that is, $\omega^{n}=1$.

\section{Circle structure arising from cyclic matrices}

In this section we consider a circle structure that always arises from a cyclic matrix which may or may not be given in terms of observations. Let the matrix entries be given in real numbers. From definition (13), we have the following lemmas immediately. Proofs are omitted.

\section{Lemma 1}

a) When $C$ is cyclic, then so are $C^{\prime}$ and its Hadmard product matrix $C^{(p)}=\left(c_{j k}^{p}\right)$ where $p$ is a positive integer.

b) When both $C_{1}$ and $C_{2}$ are cyclic, then so is their linear combination $\alpha C_{1}+\beta C_{2}$.

c) When $C$ is cyclic, then $C$ is normal, that is, $C C^{\prime}=C^{\prime} C$.

Given an asymmetric matrix $O$ that is cyclic, we construct a symmetric matrix $T$, a skew-symmetric matrix $S$ and an Hermitian matrix $H$ by (1), (2) and (3), respectively. By Lemma $1 \mathrm{a}$ and $1 \mathrm{~b}$, we see that these matrices are all cyclic . Write the eigenequation of $O$ as

$$
O z=\alpha z
$$


In view of the properties of a cyclic matrix, we find for this pair of $(\alpha, z)$ that

$$
O^{\prime} z^{*}=\alpha z^{*}
$$

Here $\boldsymbol{z}^{*}$ is the complex conjugate of $\boldsymbol{z}$. From (15) and (16) we have

$$
\begin{gathered}
T \boldsymbol{z}=\beta \boldsymbol{z} \quad \text { where } \quad \beta=\operatorname{Re}(\alpha) \\
S \boldsymbol{z}=i \gamma \boldsymbol{z} \quad \text { where } \quad \gamma=\operatorname{Im}(\alpha) .
\end{gathered}
$$

It follows from (17) and (18) that

$$
H z=\lambda z \text { where } \lambda=\beta-\gamma .
$$

The sign of real $\lambda$ may be positive, negative or even zero. It means that $H$ is generally an indefinite matrix. Applying the argument of Chino and Shiraiawa, a metric structure is not always defined in the space associated with (7) and (8) for $H$, which is constructed from a cyclic matrix.

By Lemma 1c, $C$ is normal. From the theory of linear algebra, we find out that a real cyclic matrix $C$ is diagonalized by using a real orthogonal matrix. Applying this to $S$, through some manipulation, we see that the set of singular vectors of $S$ are equivalent to the set of eigenvectors of (18).

Write the identical value of the row sum of $C$ as $c$. Matrix $C$ has an eigenvector $\mathbf{1}=(1,1, \ldots, 1)^{\prime}$ associated with the eigenvalue $c$. Setting aside such an eigenvector, we are interested only in the complex eigenvector $\boldsymbol{z}=\boldsymbol{x}+i \cdot \boldsymbol{y}$. Let $\widetilde{\boldsymbol{z}}_{j}=\left(x_{j}, y_{j}\right)^{\prime}$. Consider making a plot of $n$ points in terms of $z$ (or $z^{*}$ ), which means plotting them on a real $x-y$ plane. According to Berlin and Kac's theorem, we know that in each of those plots, the $n$ points are equally spaced on a circle (i.e. with equal arcs). But the points may be positioned differently in order on each circle. Hence, except for that ordering, the configurations are all the same. This result holds with whatever entries are in the original matrix $O$. As long as one considers metric (8) based on (7), when $H$ is not psd, it is not justified to discuss interpoint distance in the configuration. If one does not adopt the metric, then one may define the length of $\widetilde{\boldsymbol{z}}_{j}$ by the same arugument in the case of SSA. For a particular case of circle structure, equal arcs are equivalent to the equal interpoint distances.

Lemma 2 When $C=\left(c_{j k}\right)$ is cyclic and skew-symmetric, it has the following properties.

a)

$$
\sum_{k=1}^{n} c_{j k}=\sum_{k=1}^{n} c_{k j}=0 \quad(j=1,2, \ldots, n) .
$$

b) $C$ is determined in terms of $[(n-1) / 2]$ parameters at most.

c) $\operatorname{rank}(C)=2 \cdot[(n-1) / 2]$.

Proof. From (13) and by definition of skew-symmetry, we see first that $q_{n}=q_{0}=0$ because $c_{j j}=0$, and next that

$$
c_{j k}+c_{k j}=q_{n-j+k}+q_{n-k+j}=0 .
$$


Setting $j=1$ in this relation and using that $q_{n-1+k}=q_{k-1}$, we have

$$
q_{k-1}+q_{n-(k-1)}=0 \quad(k=1,2, \ldots, n) .
$$

For the case of an odd number $n=2 \ell+1$ we have $q_{0}=0$, and for the case of an even number $n=2 \ell$ we have $q_{0}=q_{\ell}=0$. For either case there are $m$ independent conditions of (21), hence we obtain (20). For both cases, we see that there are $[(n-1) / 2]$ parameters at most to determine $C$. Since the rank of a skew-symmetric matrix is always even, the rank of a cyclic and skew-symmetric matrix should be $2[(n-1) / 2]$ by $(20)$.

As mentioned above, if a skew-symmetrc matrix is cyclic, then applying SVD to it we have the circle structure with equal arcs for each pair of singular vectors. Conversely, if the SVD of a skew-symmetric matrix yields the circle structure with equal arcs for each pair of singular vectors, then it is cyclic as shown below.

Theorem 1 If every pair of singular vectors of a skew-symmetric $S$ yields the circle structure with equal arcs, then $S$ is a cyclic matrix.

Proof. Suppose that a pair of $\boldsymbol{u}=\left(u_{i}\right)$ and $\boldsymbol{v}=\left(v_{i}\right)$ associated with $\mu$ yields a circle structure with radius $r$. From (12)

$$
2=\sum_{i=1}^{n}\left(u_{i}^{2}+v_{i}^{2}\right)=n r^{2}
$$

which leads to $r^{2}=2 / n$. By the condition of equal arcs for $n$ points, the elements are written as

$$
\begin{gathered}
u_{i}=r \cos (i-1) \theta \\
v_{i}=r \sin (i-1) \theta
\end{gathered}
$$

where

$$
\theta=2 \pi / n \text { and } r=\sqrt{2 / n}
$$

Define

$$
W=\left(w_{i j}\right)=\left(\boldsymbol{u} \boldsymbol{v}^{\prime}-\boldsymbol{v} \boldsymbol{u}^{\prime}\right) .
$$

By a simple manipulation, we see that

$$
w_{i j}=u_{i} v_{j}-v_{i} u_{j}=\frac{2}{n} \sin (j-i) \theta .
$$

Noting that $\sin (2 \pi-\phi)=-\sin \phi$, we find that $W$ is the cyclic matrix satisfying (13). Suppose that the pair of $\boldsymbol{u}_{k}=\left(u_{i k}\right)$ and $\boldsymbol{v}_{k}=\left(v_{i k}\right)$ associated with $\mu_{k}$ gives a circle structure with equal arcs. The entire set of $\left\{u_{i k}\right\}$ is equal to $\left\{u_{i}\right\}$, but the ordering of those elements in $\boldsymbol{u}_{k}$ is different from that in $\boldsymbol{u}$. The same argument holds between $\boldsymbol{v}_{k}$ and $\boldsymbol{v}$. Accordingly,

$$
W_{k}=\left(\boldsymbol{u}_{k} \boldsymbol{v}_{k}^{\prime}-\boldsymbol{v}_{k} \boldsymbol{u}_{k}^{\prime}\right)
$$

is cyclic. Therefore

$$
S=\sum_{k=1}^{m} \mu_{k} W_{k}
$$


is cyclic by Lemma $1 \mathrm{~b}$.

When the original data matrix $O$ is asymmetric and cyclic, both $O$ and $S$ defined by (2) yield the same circle structures with equal arcs. Then the data analysis through SVD of $S$, if performed, reveals no practical information involved in observations. Write this sort of data matrix as $O_{1}$. However there can be asymmetric and non-cyclic $O$ from which construction of $S$ by (2) results in a cyclic matrix, as indicated below. For the sort of $O$, which we denote by $\mathrm{O}_{2}$, we again know the circle structure with equal arcs involved in $S$ without doing SVD. But the observation matrix $O_{2}$ is a little more informative than $O_{1}$ because the circle structure is found not by looking at the matrix pattern of $\mathrm{O}_{2}$ but by constructing $S$ from $\mathrm{O}_{2}$.

Theorem 2 Let $O$ be an asymmetric matrix. If the skew-symmetric $S$ defined by (2) is cyclic, then it is necessary that

$$
\sum_{k=1}^{n} o_{j k}=\sum_{k=1}^{n} o_{k j} \quad(j=1,2, \ldots, n) .
$$

Proof. If skew-symmetric $S=\left(O-O^{\prime}\right) / 2$ is cyclic, we find by Lemma 2 a that

$$
\sum_{k=1}^{n} s_{j k}=\sum_{k=1}^{n}\left(o_{j k}-o_{k j}\right)=0 \quad(j=1,2, \ldots, n),
$$

from which follows (24).

\section{Numerical illustration}

Table 1 gives an asymmetic matrix $O$ that is not cyclic but meets (24). Table 2 shows the skew-symmetric and cyclic matrix $S$ that is constructed by (2) from $O$. Although (24) is not a sufficient condition, Figure 2 represents a circle structure of five points with equal arcs, which is derived in terms of the first pair of singular vectors.

Table 1: Non-cyclic asymmetric matrix

\begin{tabular}{llllll}
\hline & 1 & 2 & 3 & 4 & 5 \\
\hline 1 & 1 & 2 & 3 & 4 & 2 \\
2 & 1 & 2 & 4 & 1 & 2 \\
3 & 5 & 3 & 3 & 2 & 0 \\
4 & 2 & 3 & 1 & 4 & 2 \\
5 & 3 & 0 & 2 & 1 & 5 \\
\hline
\end{tabular}

Table 2: Cyclic skew-symmetric matrix

\begin{tabular}{rrrrrr}
\hline & 1 & 2 & 3 & 4 & 5 \\
\hline 1 & 0.0 & 0.5 & -1.0 & 1.0 & -0.5 \\
2 & -0.5 & 0.0 & 0.5 & -1.0 & 1.0 \\
3 & 1.0 & -0.5 & 0.0 & 0.5 & -1.0 \\
4 & -1.0 & 1.0 & -0.5 & 0.0 & 0.5 \\
5 & 0.5 & -1.0 & 1.0 & -0.5 & 0.0 \\
\hline
\end{tabular}




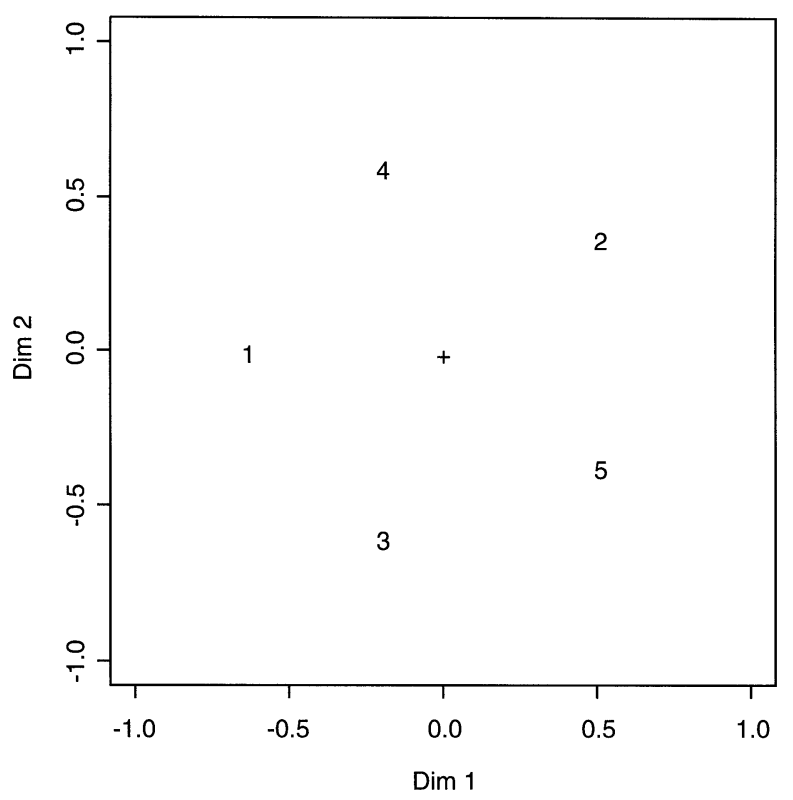

Fig. 2: The circle structure derived from the matrix of Table 2

\section{Circle structure derived from asymmetric matrices}

We have so far dealt with cyclic matrices which may be provided as original data otherwise be constructed from them. From the cyclic form, we know that those matrices involve circle structures with equal arcs. Then this type of structure may not be of principal concern for data analysis. Here we are interested in circle structures that are not determined by the matrix pattern but by the entries. From this point of view, let us examine some conditions for skew-symmetric matrices that yield circle structures on which points may or may not be equally spaced.

Theorem 3 If every pair of singular vectors of $S$ yields circle structure, then

$$
\sum_{j=1}^{n} s_{i j}^{2}=K(\text { const }) \quad(i=1,2, \ldots, n)
$$

where

$$
K=-\frac{1}{n} \operatorname{tr}\left(S^{2}\right)=\frac{2}{n} \sum_{k=1}^{m} \mu_{k}^{2}
$$

and their radii take an identical value of $\sqrt{2 / n}$. 
Proof. Let $\boldsymbol{u}_{k}=\left(u_{k i}\right)$ and $\boldsymbol{v}_{k}=\left(v_{k i}\right)$ be singular vectors associated with $\mu_{k}(k=1,2, \ldots, m)$. Using (12), we have from (11) for row $i(=1,2, \ldots, n)$,

$$
\sum_{j=1}^{n} s_{i j}^{2}=\mu_{1}^{2}\left(u_{i 1}^{2}+v_{i 1}^{2}\right)+\mu_{2}^{2}\left(u_{i 2}^{2}+v_{i 2}^{2}\right)+\cdots+\mu_{m}^{2}\left(u_{i m}^{2}+v_{i m}^{2}\right) .
$$

Assume that the k-th pair $\left(\boldsymbol{u}_{k}, \boldsymbol{v}_{k}\right)$ yields a circle structure with radius $r_{k}$. Then we have

$$
u_{i k}^{2}+v_{i k}^{2}=r_{k}^{2} \quad(i=1,2, \ldots, n ; k=1,2, \ldots, m) .
$$

Substituting this into (27) gives

$$
\sum_{j=1}^{n} s_{i j}^{2}=\sum_{k=1}^{m} \mu_{k}^{2} r_{k}^{2}=\text { const } \quad(i=1,2, \ldots, n) .
$$

Then we obtain (25), from which follows that

$$
n K=\sum_{i=1}^{n} \sum_{j=1}^{n} s_{i j}^{2}=-\operatorname{tr}\left(S^{2}\right) .
$$

In view of the property of SVD of $S$, the second term is expressed as

$$
\sum_{i=1}^{n} \sum_{j=1}^{n} s_{i j}^{2}=2 \sum_{k=1}^{m} \mu_{k}^{2}
$$

Thus we obtain (26).

When (28) holds, we see, according to the same argument for (22), that $r_{k}^{2}=2 / n(k=$ $1,2, \ldots, m)$. Accordingly each radius is identical.

Thus (25) is necessary for all the points to be located on a circle in all the twodimensional plots. Write $S^{(2)}=\left(s_{i j}^{2}\right)$. Condition (25) indicates that symmetric $S^{(2)}$ has an eigenvector of $\mathbf{1}$ associated with the eigenvalue of $K$. becomes

If $\operatorname{rank}(S)=2$ in particular, we have only one pair of singular vectors. Then (27)

$$
\sum_{j=1}^{n} s_{i j}^{2}=\mu^{2}\left(u_{i}^{2}+v_{i}^{2}\right) \quad(i=1,2, \ldots, n) .
$$

If (25) holds, it implies that all the points are located on a circle. Therefore (25) is necessary and sufficient for $S$ to yield the circle structure in the case of rank 2 .

Now consider a pair of $\boldsymbol{u}=\left(u_{i}\right)$ and $\boldsymbol{v}=\left(v_{i}\right)$ associated with $\mu$, which meets (12). Let us examine some conditions which are satisfied by $\boldsymbol{u}$ and $\boldsymbol{v}$. Define $W=(\boldsymbol{u}, \boldsymbol{v})$ and $B=\left(b_{i j}\right)=W W^{\prime}$. Noting the form that

$$
B=\boldsymbol{u} \boldsymbol{u}^{\prime}+\boldsymbol{v} \boldsymbol{v}^{\prime}
$$

we find that

$$
\sum_{i=1}^{n} \sum_{j=1}^{n} b_{i j}^{2}=\operatorname{tr}\left(B^{2}\right)=\operatorname{tr}\left(W W^{\prime} W W^{\prime}\right)=2
$$


Let

$$
\rho_{i}=b_{i i}=u_{i}^{2}+v_{i}^{2} \quad(i=1,2, \ldots, n)
$$

and denote their mean by $\bar{\rho}$. In view of (12), we see

$$
\sum_{i=1}^{n} \rho_{i}=2 \quad \text { and } \quad \bar{\rho}=2 / n .
$$

Then we have

$$
\sum_{i=1}^{n}\left(\rho_{i}-\bar{\rho}\right)^{2}=\sum_{i=1}^{n} \rho_{i}^{2}-4 / n .
$$

Since the left-hand side is nonnegative,

$$
\sum_{i=1}^{n} \rho_{i}^{2} \geq 4 / n
$$

Hence we have

$$
\sum_{i=1}^{n} b_{i i}^{2}=\sum_{i=1}^{n} \rho_{i}^{2}=\sum_{i=1}^{n}\left(u_{i}^{2}+v_{i}^{2}\right)^{2} \geq 4 / n .
$$

Combining this with (29) yields

$$
4 / n \leq \sum_{i=1}^{n}\left(u_{i}^{2}+v_{i}^{2}\right)^{2} \leq 2 .
$$

Theorem 4 If a pair of singular vectors $\boldsymbol{u}$ and $\boldsymbol{v}$ satisfies

$$
\sum_{i=1}^{n}\left(u_{i}^{2}+v_{i}^{2}\right)^{2}=4 / n
$$

then it yields the circle structure with radius $\sqrt{2 / n}$.

Proof. When (32) holds, the right-hand side of (30) becomes zero, from which follows that $\rho_{i}=2 / n$. Thus

$$
u_{i}^{2}+v_{i}^{2}=2 / n \quad(i=1,2, \ldots, n),
$$

showing a circle structure with $\sqrt{2 / n}$.

\section{Numerical illustration}

Table 3 gives a matrix of artificial data, from which follows the skew-symmetric matrix shown by Table 4. It is a non-cyclic matrix that satisfies (25), a necessary condition to yield the circle structure. Table 5 represents the singular vectors and the singular values. Each pair of singular vectors satisfies (32), a sufficient condition to yield the circle structure. In Figure 3 cases (a), (b) and (c) show 2-dimesional plots of six objects for the first, the second and the third pair of singular vectors respectively. Each reveals the circle structure of radius $1 / 3(=2 / n)$ with unequal arcs. 
Circle Structure Derived from Decomposition of Asymmetric Data Matrix

Table 3: Non-cyclic asymmetric matrix

\begin{tabular}{ccccccc}
\hline & 1 & 2 & 3 & 4 & 5 & 6 \\
\hline 1 & 0.0000 & 2.0000 & 0.1340 & 2.0000 & 1.7071 & 1.7071 \\
2 & 3.0000 & 0.0000 & 0.0000 & 1.8660 & 1.7071 & 1.7071 \\
3 & 1.8660 & 2.0000 & 0.0000 & 3.0000 & 1.7071 & 0.2929 \\
4 & 0.0000 & 0.1340 & 4.0000 & 0.0000 & 0.2929 & 1.7071 \\
5 & 0.2929 & 0.2929 & 0.2929 & 1.7071 & 0.0000 & 3.0000 \\
6 & 0.2929 & 0.2929 & 1.7071 & 0.2929 & 1.0000 & 0.0000 \\
\hline
\end{tabular}

Table 4: Non-cyclic skew-symmetric matrix

\begin{tabular}{rrrrrrrrc}
\hline & 1 & 2 & 3 & 4 & 5 & 6 & sum & sq.sum \\
\hline 1 & 0.0000 & -0.5000 & -0.8660 & 1.0000 & 0.7071 & 0.7071 & 1.0482 & 3 \\
2 & 0.5000 & 0.0000 & -1.0000 & 0.8660 & 0.7071 & 0.7071 & 1.7802 & 3 \\
3 & 0.8660 & 1.0000 & 0.0000 & -0.5000 & 0.7071 & -0.7071 & 1.3660 & 3 \\
4 & -1.0000 & -0.8660 & 0.5000 & 0.0000 & -0.7071 & 0.7071 & -1.3660 & 3 \\
5 & -0.7071 & -0.7071 & -0.7071 & 0.7071 & 0.0000 & 1.0000 & -0.4142 & 3 \\
6 & -0.7071 & -0.7071 & 0.7071 & -0.7071 & -1.0000 & 0.0000 & -2.4142 & 3 \\
\hline
\end{tabular}

Table 5: Singular value decomposition

\begin{tabular}{lrrrrrr}
\hline \multicolumn{3}{c}{ singular values } \\
\multicolumn{7}{c}{2.9696} \\
\multicolumn{3}{c}{ singular vectors } \\
\multicolumn{2}{c}{1} & 2 & 3 & 4 & 5 & 6 \\
\hline 1 & -0.5774 & -0.0000 & -0.0109 & 0.5772 & 0.5768 & 0.0244 \\
2 & -0.5425 & 0.1975 & 0.3794 & -0.4352 & -0.0761 & -0.5723 \\
3 & 0.1975 & 0.5425 & -0.4352 & -0.3794 & 0.5723 & -0.0761 \\
4 & 0.0000 & -0.5774 & -0.5772 & -0.0109 & 0.0244 & -0.5768 \\
5 & -0.4729 & -0.3312 & -0.2341 & -0.5278 & 0.0259 & 0.5768 \\
6 & 0.3312 & -0.4729 & 0.5278 & -0.2341 & 0.5768 & -0.0259 \\
\hline
\end{tabular}


SAITO

Case(a)

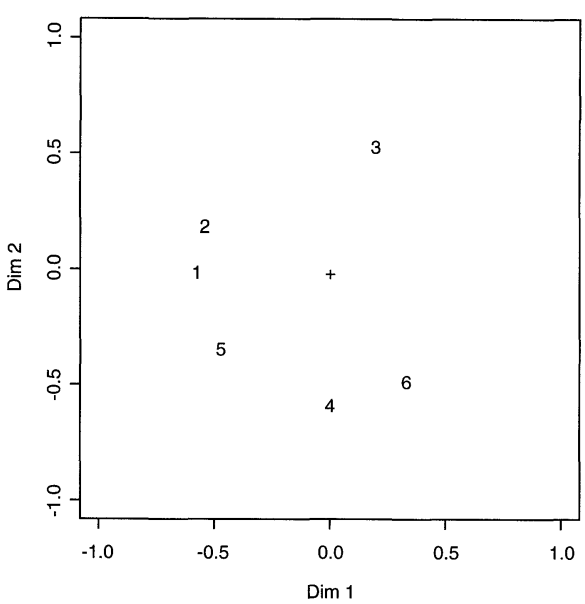

Case(b)

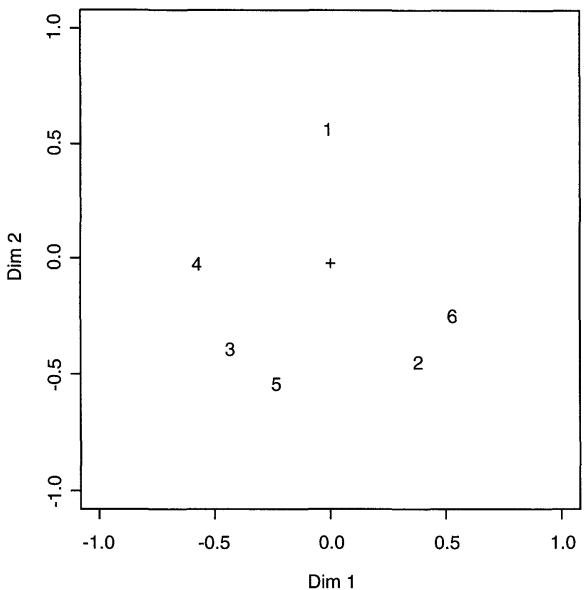

Case(c)

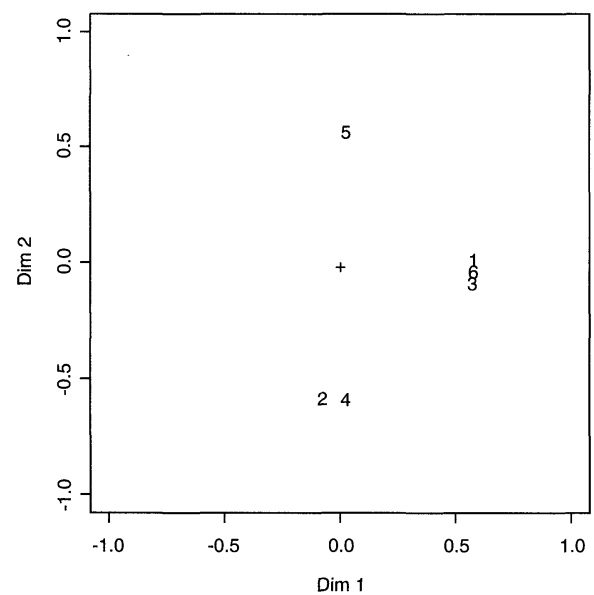

Fig. 3: The configuration of six points derived from the matrix of Table 4 


\section{Discussion}

We are in a position to discuss the entire relationship among conditions so far presented. Compare the two classes of matrices, asymmetric matrices that are cyclic and those for which $S$ defined by (2) give cyclic matrices. Both classes yield necessarily the circle structure with equal arcs. Condition (24) is necessary for the matrices of the second to yield circle structure. It will not be of interest to perform data analysis through SVD on these two classes of matrices, because the circle structure does not depend on the matrix entries but on the cyclic pattern. There would be cases in which the pattern means a siginificant property of the phenomenon that provided the data.

Theorem 3 shows a necessary condition for skew-symmetric matrices to yield circle structure of which arcs may or may not be equal. Comparing theorem 2 with theorem 3 , we note that definition (2) is assumed for $S$ in theorem 2 whereas no particular type of construction of $S$ is assumed for theorem 3. So, the interest of skew-symmetry analysis will be in data matrices that satisfy (25). If we could derive a sufficient condition for $S$ to yield the circle structure, it would be of great interest to data analysis because we know the structure inherent in data without SVD. The sufficiency remains to be investigated.

Condition (32) might be regarded as a sufficient condition for a pair of singular vectors to represent the circle structure. However it is stated implicitly in terms of the singular vectors. Conditions stated explicitly in terms of $S$ or $O$, if derived, would be more useful.

Let us discuss the basic difference in the arguments for circle structures arising from approaches of SSA and HFM. As reviewed in section 2, HFM is concerned with both $T$ and $S$ by treating $H=T+i \cdot S$, whereas SSA is so only with $S$. This difference arises from that the circle structure in using SSA is argued in the descriptive way, whereas HFM argues the structure in a metric space. For example, consider two matrices, such as $O=T+S$ and $O_{1}=T_{1}+S$ where $T \neq T_{1}$, and define $H$ and $H_{1}$ by (3). Applying SSA to $O$ and $O_{1}$ yields the same pattern in terms of singular vectors of $S$. In contrast, since $H \neq H_{1}$, applying HFM to those matrices gives generally different results. There may be a case in which the metric structure is defined for psd $H$ but it is not for indefinite $H_{1}$. Even if both $H$ and $H_{1}$ are psd, structures derived from each matrix will generally be different.

In order to examine this point further, consider two matrices of artificial data, $A$ and $B$ given in Table 6 . For example, $A$ might illustrate a situation of brand switching where off-diagonal elements indicate the switching and diagonal ones the loyality. Matrix $B$ might

Table 6: Matrices A and B

\begin{tabular}{|c|c|c|c|c|c|c|c|c|c|c|c|}
\hline \multicolumn{4}{|c|}{$\bar{A}$} & \multicolumn{4}{|c|}{$S_{(A)}$} & \multicolumn{4}{|c|}{$H_{(A)}$} \\
\hline 5 & 2 & 1 & 4 & 0 & -1 & 0 & 1 & 5 & $3-i$ & 1 & $3+i$ \\
\hline 4 & 5 & 2 & 1 & 1 & 0 & -1 & 0 & $3+i$ & 5 & $3-i$ & 1 \\
\hline 1 & 4 & 5 & 2 & 0 & 1 & 0 & -1 & 1 & $3+i$ & 5 & $3-i$ \\
\hline 2 & 1 & 4 & 5 & -1 & 0 & 1 & 0 & $3-i$ & 1 & $3+i$ & 5 \\
\hline \multicolumn{4}{|c|}{ B } & \multicolumn{4}{|c|}{$S_{(B)}$} & \multicolumn{4}{|c|}{$H_{(B)}$} \\
\hline 0 & 2 & 1 & 4 & \multirow{4}{*}{\multicolumn{4}{|c|}{ same as above }} & 0 & $3-i$ & 1 & $3+i$ \\
\hline 4 & 0 & 2 & 1 & & & & & $3+i$ & 0 & $3-i$ & 1 \\
\hline 1 & 4 & 0 & 2 & & & & & 1 & $3+i$ & 0 & $3-i$ \\
\hline 2 & 1 & 4 & 0 & & & & & $3-i$ & 1 & $3+i$ & 0 \\
\hline
\end{tabular}


Table 7: Eigenvalues and eigenvectors

\begin{tabular}{cccccc}
\hline \multirow{4}{*}{$H_{(A)}$} & $\lambda$ & 12 & 6 & 2 & 0 \\
\cline { 2 - 6 } & & 0.5 & $-0.5 i$ & $0.5 i$ & 0.5 \\
& & 0.5 & 0.5 & 0.5 & -0.5 \\
& & 0.5 & $0.5 i$ & $-0.5 i$ & 0.5 \\
& & 0.5 & -0.5 & -0.5 & -0.5 \\
\hline \multirow{4}{*}{$H_{(B)}$} & & 7 & 1 & -3 & -5 \\
\cline { 2 - 6 } & & 0.5 & $-0.5 i$ & $0.5 i$ & 0.5 \\
& & 0.5 & 0.5 & 0.5 & -0.5 \\
& & 0.5 & $0.5 i$ & $-0.5 i$ & 0.5 \\
\hline
\end{tabular}

represent pecking frequencies observed among pigeons (or any behavior of competition), where individual $j$ pecks individual $k(\neq j)$ but never does itself. Then diagonal entries are naturally zero by virtue of the behavior, accordingly, it does not make sense to estimate them. This situation should strictly be differentiated from another in which estimating some missing entries of diagonal elements may be justified.

Write matrices associated with $A$ or $B$ as $H_{(A)}, S_{(A)}, H_{(B)}, S_{(B)}$, respectively. We see that $A, B$ and the matrices associated with them are all cyclic. Thus, according to Berlin and Kac's theorem, we know that either of these matrix yields the circle structure in the plot in terms of a complex eigenvector or in the plot in terms of a pair of singular vectors. Table 7 shows solutions of (5), the eigenvalues and eigenvectors of $H_{(A)}$ and those of $H_{(B)}$. We note that $H_{(A)}$ and $H_{(B)}$ have the same eigenvectors, just as described by Berlin and Kac's theorem. Denote them by $\boldsymbol{z}_{j}$ associated with the $j$-th eigenvalue $(j=1, \ldots, 4)$. Given either $A$ or $B$, plotting objects in terms of $z_{2}$ or $z_{3}$ reveals the identical pattern of circle. It is found that $H_{(B)}$ is indefinite by property (6) whereas $H_{(A)}$ is psd as illustrated in Table 7. Therefore, according to Chino and Shiraiwa' argument, even for the visually identical pattern for both cases, the metric structure can be defined in the case of $A$ but cannot be in the case of $B$. (From (9) we see that $\left\|\widetilde{\boldsymbol{w}}_{j}\right\|=0 ; j=1, \ldots, 4$ for $B$.) When it is of principal concern in analysis of asymmetric data matrix to argue the structure in the metric space defined by (7) and (8), if one neglects the definiteness of a matrix and discuss the pattern in the two-dimensional plot for each $\boldsymbol{z}_{j}$, that would be misleading.

\section{Concluding remarks}

For analysis of asymetric data matrices, our focus of interest has been on the standpoint of descriptive data analysis in a way similar to the biplot display of multivariate data (Gabriel, 1981). When we take Gower's approach using SVD of the skew-symmetric matrix constructed from a data matrix, we aim to detect some information involved in the data by examining any pattern in the two-dimensional plot in terms of a pair of singular vectors. From this standpoint, we are interested in the circle structure appearing in such a plot, and presented some algebraic conditions under which circle structure appears through the SVD. As reviewed in the preliminaries and stated clearly above, the argument of strucrure for this purpose should be differentiated basically from the argument of structure in a metric vector space.

Turning back to our definition of the study, we see that the data will often involve ran- 
dom variation or observation error. Then we would encounter cases in which some conditions of necessity or sufficiency for infallible data would not hold exactly but do approximately. Assuming asymmetric data randomly sampled from a population of objects, in which any structure might be hidden in the asymmetric relationships, the results presented in this paper seem to serve as a basis to examine a hypothetical structure.

\section{Acknowledgements}

An earlier version of this paper was presented at the joint Annual Meeting of the Psychometric Society and the Classification Society of North America, University of Illinois, 1998, which appeared later in Saito (1998). The author wishes to thank P.J.F. Groenen for his comments at the early stage of the present study. Comments from an anonymous referee, claiming the precedence of HFM over SSA, served in the end to clarify the differences between the two approaches regarding the distance definitions, so that such a claim might never be posed. The author greatly appreciates kind advice from the editor.

\section{REFERENCES}

Berlin, T.H. and Kac, M. (1952). The spherical model of a ferromagnet. Physical Review 86, 821-835.

Bock, R.D. and Jones, L.V. (1968). The Measurement and Prediction of Judgment and Choice. San Francisco: Holden-Day.

Chino, N. (1978). A graphical technique for representing the asymmetric relationships between N objects. Behaviormetrika 5, 23-40.

Chino, N. and Shiraiwa, K. (1993). Geometric structures of some non-distance models for asymmetric MDS. Behaviormetrika 20, 35-47.

Constantine, A.G. and Gower, J.C. (1978). Graphical representation of asymmetric matrices. Applied Statistics 27, 297-304.

Escoufier, Y. and Grorud, A. (1980). Analyse factorielle des matrices carrees non-symétriques. In Data Analysis and Informatics, E. Diday (ed.), 263-276, New York: NorthHolland.

Ferligoj, A. and Batagelj, V. (1983). Some types of clustering with relational constraints. Psychometrika 48, 541-552.

Gabriel, K.R. (1981). Biplot display of multivariate matrices for inspection of data and diagnosis. In Interpreting Multivariate Data, V. Barnett (ed.), 147-173. Chichester: Wiley.

Gower, J.C. (1977). The analysis of asymmetry and orthogonality. In Recent Developments in Statistics, J.R. Barra et al. (eds.), 109-123, New York: North-Holland.

Harshman, R.A. (1978). Models for analysis of asymmetrical relationships among $N$ objects or stimuli. Paper presented at the First Joint Meeting of the Psychometric Society and the Society of Mathematical Psychology, Hamilton, Ontario.

Harshman, R.A., Green, P.E., Wind, Y. and Lundy, M.E. (1982). A model for the analysis of asymmetric data in marketing research. Marketing Science 1, 205-242.

Hobbs, R.J. and Legg, C.J. (1984). Markov models and initial floristic composition in heathland vegetation dynamics. Vegetation 56, 31-43.

Saito, T. (1991). Analysis of asymmetric proximity matrix by a model of distance and additive terms. Behaviormetrika 29, 45-60. 
Saito, T. (1994). Psychological scaling of the asymmetry observed in comparative judgement. British Journal of Mathematical and Statistical Psychology 47, 41-62.

Saito, T. (1997). Line structure derived from decomposition of asymmetric matrix. Journal of the Japanese Society of Computer Statistics 10, 47-57.

Saito, T. (1998). Typical patterns inherent in asymmetric data matrices. Valdes Research Paper, Series E, No. 2.

Sato, Y. (1988). An analysis of sociometric data by MDS in Minkowski space. Statistical Theory and Data Analysis II. K. Matsusita (ed.), Amsterdam: North-Holland, 385-396.

Sato, Y. (1989). On a distance representation of asymmetric dissimilarities by Minkowski metric function. Bulletin of the Computational Statistics of Japan 2, 35-45. (in Japanese)

Weeks, D.G. and Bentler, P.M. (1982). Restricted multidimensional scaling model for nonsymmetric proximities. Psychometrika 47, 201-208.

(Received December 1999, Accepted April 2002) 